Pacific Journal of Mathematic 


\title{
SELF-ADJOINT DIFFERENTIAL OPERATORS
}

\author{
ARNold L. Villone
}

Let $\mathscr{H}$ denote the Hilbert space of square summable analytic functions on the unit disk, and consider the formal differential operator

$$
L=\sum_{i=0}^{n} p_{i} D^{i}
$$

where the $p_{i}$ are in $\mathscr{H}$. This paper is devoted to a study of symmetric operators in $\mathscr{H}$ arising from $L$. A characterization of those $L$ which give rise to symmetric operators $S$ is obtained, and the question of when such an $S$ is selfadjoint or admits of a self-adjoint extension is considered. If $A$ is a self adjoint extension of $S$ and $E(\lambda)$ the associated resolution of the identity, the projection $E_{\Delta}$ corresponding to the interval $\Delta=(a, b]$ is shown to be an integral operator whose kernel can be expressed in terms of a basis of solutions for the equation $(L-\ell) u=0$ and a spectral matrix.

Let $\mathscr{A}$ denote the space of functions analytic on the unit disk and $\mathscr{C}$ the subspace of square summable functions in $\mathscr{A}$ with inner product

$$
(f, g)=\iint_{|z|<1} f(z) \overline{g(z)} d x d y
$$

Then $\mathscr{H}$ is a Hilbert space with the reproducing property, i.e., for each $z$ there exists a unique element $K_{z}$ of $\mathscr{\mathscr { C }}$ such that

$$
f(z)=\left(f, K_{z}\right) \text {. }
$$

Moreover, if the sequence $\left\{f_{n}\right\}$ converges to $f$ in norm, $f_{n}(z)$ converges to $f(z)$ uniformly on compact subsets of the disk. A complete orthonormal set for $\mathscr{H}$ is provided by the normalized powers of $z$,

$$
e_{n}(z)=[(n+1) / \pi]^{1 / 2} z^{n}, n=0,1, \cdots .
$$

From this it follows that $\mathscr{H}$ is identical with the space of power series $\sum_{n=0}^{\infty} a_{n} z^{n}$ which satisfy

$$
\sum_{n=0}^{\infty}\left|a_{n}\right|^{2} /(n+1)<\infty
$$

Consider the formal differential operator

$$
L=p_{n} D^{n}+\cdots+p_{1} D+p_{0},
$$


where $D=d / d z$ and the $p_{i}$ are in $\mathscr{A}$. For $f$ in $\mathscr{L}$ the element $L f$ is in $\mathscr{A}$, but not necessarily in $\mathscr{C}$. To see this we take $L=d / d z$ and $f(z)=\sum_{n=1}^{\infty} n^{-1 / 2} z^{n}$, from (1.1) it follows that $f$ is in $\mathscr{H}$ but $L f$ is not. In order to consider $L$ as an operator in $\mathscr{H}$ we must restrict the class of functions on which $L$ acts in some suitable manner. Since our concern is with densely defined operators it is only natural to demand that powers of $z$ be mapped into $\mathscr{H}$. This requires some restrictions on the coefficients of $L$. As an example consider the operator $L=p D$ where $p(z)=\sum_{n=0}^{\infty}(n+1) z^{n}$.

We have $L e_{k}(z)=k(k+1)^{1 / 2} \pi^{-1 / 2} \sum_{n=k-1}^{\infty}(n-k)^{1 / 2} z^{n}$, and hence $L e_{k} \notin \mathscr{Y}$. A sufficient condition for the $L e_{k}$ to be in $\mathscr{C}$ is that the coefficients $p_{i}$ be in $\mathscr{H}$.

Let $L=\sum_{i=0}^{n} p_{i} D^{i}$, where the $p_{i}$ are in $\mathscr{L}$, and let $\mathscr{D}_{0}$ denote the span of the $e_{k}$ and $\mathscr{D}$ the set of all $f$ in $\mathscr{C}$ for which $L f$ is in $\mathscr{C}$. We now define the operators $T_{0}$ and $T$ as follows.

$$
\begin{aligned}
T_{0} f & =L f \quad f \in \mathscr{D}_{0}, \\
T f & =L f \quad f \in \mathscr{D} .
\end{aligned}
$$

THEOREM 1.1. $T_{0}$ and $T$ are densely defined operators with range in $\mathscr{H}, T_{0} \subseteq T$, and $T$ is closed.

Proof. We first show that $T$ is closed. Let $\left\{f_{n}\right\}$ be a sequence of functions in $\mathscr{D}$ such that $f_{n} \rightarrow f$ and $T f_{n} \rightarrow g$, hence $f_{n}(z)$ and $L f_{n}(z)$ converge uniformly on compact subsets to $f(z)$ and $g(z)$ respectively. But $L f_{n}(z)$ also converges to $L f(z)$. Hence $L f(z)=g(z)$, $|z|<1$, so $T f \in \mathscr{H}$ and $T f=g$.

Since $\mathscr{D}_{0}$ is dense in $\mathscr{C}$ and $T_{0} f=T f$ for $f \in \mathscr{D}_{0} \cap \mathscr{D}$ it suffices to show that the $e_{j}$ are in $\mathscr{D}$. Since $L e_{j}=\sum_{i=0}^{n} p_{i} D^{i} e_{j}$ and $p_{i} D^{i} e_{j}$ is either zero or of the form $p_{i} e_{k}$ for some nonnegative integer $k$, it sufficies to show that $p_{i} e_{k} \in \mathscr{H}$. Let $p_{i}=\sum_{j=0}^{\infty} a_{j} e_{j}$, a simple computation yields

$$
e_{k} e_{j}=[(k+1) \pi]^{1 / 2}[(j+1) /(j+k+1)]^{1 / 2} e_{j+k},
$$

and consequently,

$$
\left\|e_{k} p_{i}\right\|^{2} \leqq[(k+1) \pi]\left\|p_{i}\right\|^{2}<\infty .
$$

$T_{0}$ and $T$ are respectively the minimal and maximal operators in $\mathscr{H}$ associated with the formal operator $L$. We now proceed to study the class of formal differential operators for which $T_{0}$ is symmetric.

It is clear that the operator $T_{0}$ associated with the formal differential operator $L$ is symmetric if and only if 


$$
\left(L e_{n}, e_{m}\right)=\left(e_{n}, L e_{m}\right), n, m=0,1, \cdots \text {. }
$$

We shall refer to those formal operators satisfying (1.2) as formally symmetric. As an example we have the real Euler operator

$$
L=\sum_{i=0}^{n} a_{i} z^{i} D^{i},
$$

$a_{i}$ real. Then $L e_{j}=p(j) e_{j}$ where $p$ is the characteristic polynomial

$$
p(x)=a_{0}+a_{1} x+\cdots+a_{n} x(x-1) \cdots(x-n+1) .
$$

Since $p(j)=\overline{p(j)}, L$ is formally symmetric. A characterization of formally symmetric $L$ in terms of the coefficients $p_{i}$ is given in the next section. We now proceed to the consideration of the adjoint operators $T_{0}^{*}$ and $T^{*}$. In what follows we shall make use of the result that if $L$ is formally symmetric of order $n$, then the coefficients $p_{i}$ are polynomials of degree at most $n+i, i=0,1, \cdots, n$. A proof of this is given in Theorem 2.2.

THEOREM 1.2. If $T_{0}$ is symmetric, $T_{0}^{*}=T$ and $T^{*} \leqq T$. The closure of $T_{0}, S$, is self adjoint if and only if $S=T$.

Proof. By Theorem 2.2 the coefficients $p_{i}$ are polynomials of degree at most $n+i$. This implies that $T_{0}$ maps $\mathscr{D}_{0}$ into itself. In particular,

$$
\begin{gathered}
L e_{m}=\sum_{i=0}^{n+m} \alpha_{i} e_{i}, \quad 0 \leqq m \leqq n, \\
L e_{n+j}=\sum_{i=j}^{2 n+j} \alpha_{i} e_{i}, \quad j=1,2, \cdots .
\end{gathered}
$$

Using this we show that $T_{0}^{*} \subseteq T$. Let $g=\sum_{j=0}^{\infty} a_{j} e_{j}$ and $g^{*}=\sum_{j=0}^{\infty} b_{j} e_{j}$ be in the graph of $T_{0}^{*}$ and consider the sequence $\left\{g_{p}\right\}$ in $\mathscr{D}_{0}$ defined as $g_{p}=\sum_{j=0}^{p} a_{j} e_{j}$. Since $g_{p} \rightarrow g$ we have $\left(T_{0} e_{k}, g_{p}\right) \rightarrow\left(T_{0} e_{k}, g\right)=\left(e_{k}, g^{*}\right)$. Hence $\left(e_{k}, T_{0} g_{p}\right) \rightarrow\left(e_{k}, g^{*}\right)$. Now $L g$ is in $\mathscr{A}$ and $T_{0} g_{p}$ converges to $L g$ uniformly on compact subsets. Since the $e_{j}$ are just the normalized powers of $z$, the power series expansion of $L g$ can be written as $\sum_{j=0}^{\infty} c_{j} e_{j}(z)$. Since $L g_{p}(z)=\sum_{j=0}^{p} a_{j} L e_{j}(z)$ converges uniformly to $\sum_{j=0}^{\infty} c_{j} e_{j}(z)$, it follows from (1.3) that $L g_{p}$ has the same coefficient of $e_{m}$ as does $L g$ for $p>n+m+1$. Hence $\left(e_{m}, T_{0} g_{p}\right)=\bar{c}_{m}$ for $p>n+m+1$ and since $\left(e_{m}, T_{0} g_{p}\right) \rightarrow\left(e_{m}, g^{*}\right)$ we have $c_{m}=b_{m}$. Therefore $g^{*}=L g$, so that $g \in \mathscr{D}$ and $g^{*}=T g$.

To show that $T \cong T_{0}^{*}$ it will suffice to show that $\left(T_{0} e_{m}, g\right)=$ $\left(e_{m}, T g\right)$ for all $g$ in $\mathscr{D}$ and $m=0,1, \ldots$ Let $g=\sum_{j=0}^{\infty} a_{j} e_{j}$ be in $\mathscr{D}$ and $g_{p}$ as before. Since $T_{0}$ is symmetric and $g_{p} \rightarrow g$ we have $\left(e_{m}, T_{0} g_{p}\right)=\left(T_{0} e_{m}, g_{p}\right) \rightarrow\left(T_{0} e_{m}, g\right)$. By precisely the same argument 
as before $\left(e_{m}, T_{0} g_{p}\right)=\left(e_{m}, T g\right)$ for $p>n+m+1$, from which it follows that $\left(e_{m}, T g\right)=\left(T_{0} e_{m}, g\right)$ and $T_{0}^{*}=T$. Since $T_{0} \subseteq T, T^{*} \subseteq T_{0}^{*}=T$.

The closure $S$ of the symmetric operator $T_{0}$ is given by $T_{0}^{* *}=$ $T^{*} \leqq T$. Since $T$ is closed $T^{* *}=T$, from which it follows that $S^{*}=T$. Hence $S=T$ implies $S=S^{*}$. Conversely if $S$ is selfadjoint we have $S=T^{*}=S^{*}=T$.

A sufficient condition for $T$ to be self-adjoint is given by the following theorem.

THEOREM 1.3. For $f=\sum_{j=0}^{\infty} a_{j} e_{j} \operatorname{set} f_{m}=\sum_{j=0}^{m} a_{j} e_{j}$. If $\sup _{m}\left\|T f_{m}\right\|<\infty$ for each $f$ in $\mathscr{D}$, then $S$ is self-adjoint.

Proof. Since $T^{*} \subseteq T, T$ symmetric implies $T=T^{*}$ and hence $S=S^{*}$. We show that $(T f, g)-(f, T g)$ vanishes for all $f, g$ in $\mathscr{D}$. If $L$ is of order $n$ we have $\left(T f_{m}, g_{p}\right)=\left(T f, g_{p}\right)$ for $m>n+p+1$. Using this fact and the symmetry of $T_{0}$ we obtain

$$
\begin{aligned}
\left(T f, g_{k n}\right) & =\left(T f_{k n+n+1}, g_{k n}\right)=\left(f_{k n+n+1}, T g_{k n}\right) \\
& =\left(f_{k n-n-1}, T g_{k n}\right)+\left(f_{k n+n+1}-f_{k n-n-1}, T g_{k n}\right) \\
& =\left(f_{k n-n-1}, T g\right)+\left(f_{k n+n+1}-f_{k n-n-1}, T g_{k n}\right) \\
& \quad k=1,2, \ldots .
\end{aligned}
$$

Therefore,

$$
(T f, g)-(f, T g)=\lim _{k \rightarrow \infty}\left(f_{k n+n+1}-f_{k n-n-1}, T g_{k n}\right) .
$$

Since the $T g_{k n}$ are bounded in norm this implies $(T f, g)-(f, T g)=0$.

COROLlaRY. If $L$ is a formally symmetric Euler operator, then $S$ is self-adjoint.

Proof. For $f=\sum_{j=0}^{\infty} b_{j} e_{j}$ in $\mathscr{D}, T f$ and $T f_{m}$ are given by $\sum_{j=0}^{\infty} p(j) b_{j} e_{j}$ and $\sum_{j=0}^{m} p(j) b_{j} e^{j}$ respectively, where $p(x)$ is the characteristic polynomial for $L$. Hence

$$
\left\|T f_{m}\right\|^{2}=\sum_{j=0}^{m} p(j)^{2}\left|b_{j}\right|^{2} \leqq\|T f\|^{2},
$$

and the result follows.

2. Formal considerations. The formal operator $L=\sum_{i=0}^{n} p_{i} D^{i}$ is formally symmetric if

$$
\left(L e_{n}, e_{m}\right)=\left(e_{n}, L e_{m}\right), n, m=0,1, \ldots .
$$

To obtain a characterization of the formally symmetric operators 
in terms of their coefficients we first determine the action of $L$ on $e_{k}$.

LEMma 2.1. Let $L=\sum_{i=0}^{n} p_{i} D^{i}$ where $p_{i}(z)=\sum_{k=0}^{\infty} a_{k}(i) z^{k}$. Then $L e_{i}=\sum_{j=0}^{\infty} c_{i j} e_{j}$ where

$$
\begin{aligned}
& c_{i j}=A(i, j) \sum_{k=0}^{n} B(i, k) a_{j-i+k}(k), \quad i, j=0,1 \cdots, \\
& A(i, j)=[(i+1) /(j+1)]^{1 / 2}, \\
& B(i, k)=i ! /(i-k) ! \quad i \geqq k \\
& =0 \quad i<k \text {. }
\end{aligned}
$$

Proof. Consider the elementary operators $L_{p q}=z^{p} D^{q}, p, q=$ $0,1, \ldots$ A simple calculation yields

$$
L_{p q} e_{m}=B(m, q) A(m, m+p-q) e_{m+p-q} .
$$

Now consider $L e_{m}$ (as an element of $\mathscr{A}$ ),

$$
\begin{aligned}
L e_{m}(z) & =\sum_{i=0}^{n} \sum_{k=0}^{\infty} a_{k}(i) L_{k i} e_{m}(z) \\
& =\sum_{i=0}^{n} \sum_{k=0}^{\infty} a_{k-m+i}(i) B(m, i) A(m, k) e_{k}(z) \\
& =\sum_{k=0}^{\infty} c_{m k} e_{k}(z) \quad|z|<1 .
\end{aligned}
$$

But $e_{k}(z)$ is just a multiple of $z^{k}$, therefore it follows from the uniqueness of power series representation of elements of $\mathscr{A}$, that $\sum_{k=0}^{\infty} c_{m k} e_{k}$ converges to $T e_{m}$ in $\mathscr{L}$.

It follows that $L$ is formally symmetric if and only if the coefficients $a_{k}(\ell), \ell, k=0,1, \cdots$, satisfy the linear system

$$
c_{i j}=\overline{c_{j i}}, \quad i, j=0,1, \cdots .
$$

The following provides a simplification of the system (2.2).

THEOREM 2.2. If $L=\sum_{i=0}^{n} p_{i} D^{i}$ is formally symmetric the $p_{i}$ are polynomials of degree at most $n+i$.

Proof. Consider $c_{n+p, 0}$ for $p \geqq 1$. Since $j-n-p<0$ for $p \geqq 1$ and $j=0, \cdots, n, a_{j-n-p}(j)=0$. Consequently $c_{n+p, 0}=\bar{c}_{0, n+p}$ reduces to $A(0, n+p) a_{n+p}(0)=0, p \geqq 1$, and $p_{0}$ is of degree at most $n$. We now proceed inductively. Consider

$$
c_{n+p, k+1}=\bar{c}_{k+1, n+p}, \quad p \geqq k+2 \text {. }
$$


Since $k+1+j-n-p<0$ for $p \geqq k+2$ and $j=0, \cdots, n$, reduces to

$$
A(k+1, n+p) \sum_{j=0}^{k-1} B(k+1, j) a_{n+p+j-k-1}(j)=0, \quad p \geqq k+2 .
$$

Since $n+p+j-k-1 \geqq n+j+1$, it follows from the inductive hypothesis that $a_{n+p+j-k-1}(j)=0$ for $j=0, \cdots, k$, and hence

$$
A(k+1, n+p)(k+1) ! a_{n+p}(k+1)=0, \quad p \geqq k+2 .
$$

Therefore degree $p_{k+1} \leqq n+k+1$.

This result allows a considerable simplification of the system (2.2). For each nonnegative integer $p$ consider the subsystem $S_{p}$ of $(2.2)$

$$
c_{i, i+p}=\bar{c}_{i+p, i}, \quad i=0,1, \cdots .
$$

Since the equation $c_{i j}=\bar{c}_{j i}$ appears only in $S_{|i-j|}$ we have a partition of (2.2). Since the $p_{i}$ are polynomial of degree at most $n+i$,

$$
a_{\ell+p}(\ell)=0 \quad p>n, \quad \ell=0, \cdots, n,
$$

from which it follows that $S_{p}$ is trivial for $p>n$. From (2.1) we see that $a_{\ell}(i)$ appears only in $S_{|\ell-i|}$. Hence (2.2) is equivalent to the $n+1$ systems,

$$
S_{p}: c_{i, i+p}=\bar{c}_{i+p, i}, \quad i=0,1, \cdots,
$$

where the $a_{j+p}(j)$ appear only in $S_{p}$. Using (2.1) this becomes

$$
S_{p}: \sum_{k=0}^{n} a_{p+k}(k) B(i, k)=\sum_{k=p}^{n} \bar{a}_{k-p}(k) B(i+p, k) A^{2}(i+p, i) .
$$

THEOREM 2.3. The system $S_{p}$ is satisfied if and only if

$$
j ! a_{j+p}(j)=R_{0}^{j} \quad j=0,1, \cdots, n,
$$

where $R_{i}^{0}=\sum_{k=p}^{n} \bar{a}_{k-p}(k) B(i+p, k) A^{2}(i+p, i)$, and the $R_{i}^{i}$ are obtained recursively by

$$
R_{i}^{\dot{i}}=R_{i+1}^{i-1}-R_{i}^{j-1}
$$

Proof. For fixed $p$ denote the left and right hand sides of the $i$ th member of $S_{p}$ by $L_{i}^{0}$ and $R_{i}^{0}$ respectively. We now employ a reduction scheme. Form the sequence of systems $\left\{L_{i}^{1}=R_{i}^{1}\right\},\left\{L_{i}^{2}=R_{i}^{2}\right\}, \cdots$, where

$$
\begin{aligned}
& L_{i}^{j+1}=L_{i+1}^{j}-L_{i}^{j} \\
& R_{i}^{j+1}=R_{i+1}^{j}-R_{i}^{j}
\end{aligned} \quad i, j=0,1, \cdots
$$


By induction on $j$ it can be shown that

$$
L_{i}^{j}=\sum_{k=0}^{n} a_{k+p}(k) B(i, k-j) P_{j}(k)
$$

where $P_{j}(k)=k(k-1) \cdots(k-j+1)$. Consequently, $L_{0}^{j}=j ! a_{j+p}(j)$ and the necessity follows.

For the sufficiency we use the fact that for a given system of linear equations, $L^{j}=R^{j}, j=0 \cdots, n$, there exists a unique set of linear systems $\left\{\hat{L}_{i}^{0}=\hat{R}_{i}^{0}\right\}, \cdots,\left\{\hat{L}_{i}^{n}=\hat{R}_{i}^{n}\right\}$ which have the properties $P 1$ thru $P 3$.

$$
\begin{array}{rlrl}
P 1 \hat{L}_{i}^{j} & =\hat{L}_{i+1}^{j-1}-\hat{L}_{i}^{j-1} & \\
\hat{R}_{i}^{j} & =\hat{R}_{i+1}^{j-1}-\hat{R}_{i}^{j-1} & & j=1, \cdots, n \\
\text { P2 } \hat{L}_{0}^{j} & =L^{j}, \hat{R}_{0}^{j}=R^{j} & & i=0,1, \cdots \\
\text { P3 } \hat{L}_{i}^{n} & =L^{n}, \hat{R}_{i}^{n}=R^{n} & & j=0, \cdots, n
\end{array}
$$

This set is constructed in the following manner.

The system $\left\{\hat{L}_{i}^{n}=\hat{R}_{i}^{n}\right\}$ is defined by $P 3$. To satisfy $P 1$ and $P 2$ we define the system $\left\{\hat{L}_{i}^{n-1}=\hat{R}_{i}^{n-1}\right\}$ inductively by $\hat{L}_{0}^{n-1}=L^{n-1}, \hat{R}_{0}^{n-1}=$ $R^{n-1}, \quad \hat{L}_{i+1}^{n-1}=\hat{L}_{i}^{n-1}+L^{n}$, and $\hat{R}_{i+1}^{n-1}=\hat{R}_{i}^{n-1}+R^{n}$. Similarly we define the system $\left\{\hat{L}_{i}^{n-2}=\hat{R}_{i}^{n-2}\right\}$ through $\left\{\hat{L}_{i}^{0}=\hat{R}_{i}^{0}\right\}$ by means of the equations

$$
\begin{aligned}
& \hat{L}_{0}^{n-2}=L^{n-2}, \hat{R}_{0}^{n-2}=R^{n-2} \\
& \hat{L}_{i+1}^{n-2}=\hat{L}_{i}^{n-2}+\hat{L}_{i}^{n-1}, \hat{R}_{\imath+1}^{n-2}=\hat{R}_{i}^{n-2}+\hat{R}_{i}^{n-1} \\
& \hat{L}_{0}^{0}=L^{0}, \hat{R}_{0}^{0}=R^{0} \\
& \hat{L}_{i+1}^{0}=\hat{L}_{i}^{0}+\hat{L}_{i}^{1}, \hat{R}_{i+1}^{0}=\hat{R}_{i}^{0}+\hat{R}_{i}^{1} .
\end{aligned}
$$

From the method of construction the systems $\left\{\hat{L}_{i}^{0}=\hat{R}_{i}^{0}\right\}$ thru $\left\{\hat{L}_{i}^{n}=\hat{R}_{i}^{n}\right\}$ are the unique systems satisfying $P 1$ thru $P 3$.

Since $P_{j}(k)$ vanishes for $0 \leqq k \leqq j-1$ it follows that $L_{i}^{j}=0$ for $j>n$ and all $i$. Moreover, for $j=n$ we have $L_{i}^{n}=n ! a_{n+p}(n)$, a constant independent of $i$. From (2.4) we see that $R_{i}^{0}=$ $\sum_{k=p}^{n} \bar{a}_{k-p}(k) C_{k}(i)$, where the $C_{k}(i)$ are polynomials in $i$ of degree $k$. Hence $R_{i}^{1}=R_{i+1}^{0}-R_{i}^{0}$ can be written in the form $\sum_{k=p}^{n} \bar{a}_{k-p}(k) C_{k}^{1}(i)$, where the $C_{k}^{1}(i)$ are of degree $k-1$. Continuing in this manner we obtain

$$
\begin{array}{ll}
R_{i}^{j}=0 \quad j>n & i=0,1, \cdots, \\
R_{i}^{n}=R_{0}^{n} & i=0,1, \cdots .
\end{array}
$$

Hence the systems $\left\{L_{i}^{j}=R_{i}^{j}\right\} j=0, \cdots, n$ satisfy $P 1$ thru $P 3$ where $L_{0}^{j}=R_{0}^{j}$ corresponds to the $L^{j}=R^{j}$ and the system $\left\{\hat{L}_{i}^{0}=\hat{R}_{i}^{0}\right\}$ corresponds to the system $S_{p}$. This yields the sufficiency.

This theorem provides an algorithm for determining all formally 
symmetric operators of a given order. As an application we give the general formally symmetric first order operator. Use of 2.5 for $p=0$ and 1 yields

$$
L=\left(c z^{2}+a z+\bar{c}\right) d / d z+(2 c z+b),
$$

where $a$ and $b$ are real.

3. Self-adjoint extensions. The operator $S$ has another characterization which will be of use in the study of self-adjoint extensions. For $f$ and $g$ in $\mathscr{D}$ consider the bilinear form

$$
\langle f g\rangle=(L f, g)-(f, L g),
$$

and let $\tilde{\mathscr{D}}$ be the set of those $f$ in $\mathscr{D}$ for which $\langle f g\rangle=0$ for all $g$ in $\mathscr{D}$. Since $S=T^{*}$ and $\mathscr{D}\left(T^{*}\right)=\tilde{\mathscr{D}}, S$ has domain $\tilde{\mathscr{D}}$.

Let $\mathscr{D}^{+}$and $\mathscr{D}^{-}$denote the set of all solutions of the equations $L u=i u$ and $L u=-i u$ respectively, which are in $\mathscr{Z}$. It is known from the general theory of Hilbert space [3, p. 1227-1230] that

$$
\mathscr{D}=\tilde{\mathscr{D}}+\mathscr{D}^{+}+\mathscr{D}^{-},
$$

and every $f \in \mathscr{D}$ has the unique representation

$$
f=\tilde{f}+f^{+}+f^{-},\left(\tilde{f} \in \tilde{\mathscr{D}}, f^{+} \in \mathscr{D}^{+}, f^{-} \in \mathscr{D}^{-}\right) .
$$

Let the dimensions of $\mathscr{D}^{+}$and $\mathscr{D}^{-}$be $m^{+}$and $m^{-}$respectively. Clearly, $m^{+}$and $m^{-}$cannot exceed the order of $L$. These integers are referred to as the deficiency indices of $S$, and $S$ has self-adjoint extensions if and only if $m^{+}=m^{-}$. Moreover $S$ is itself self-adjoint if and only if $m^{+}=m^{-}=0$.

We assume that $m^{+}=m^{-}=m$ and seek to characterize all selfadjoint extensions of $S$. Von Neumann has shown that the selfadjoint extensions of $S$ are in a one-to-one correspondence with the unitary operators $U$ of $\mathscr{D}^{+}$onto $\mathscr{D}^{-}$. Corresponding to any such $U$ there exists a self-adjoint extension $A$ of $S$ whose domain is the set of all $f \in \mathscr{D}$ which are of the form

$$
f=\tilde{f}+(I-U) f^{+}, \quad\left(f \in \tilde{\mathscr{D}}, f^{+} \in \mathscr{D}^{+}\right),
$$

where $I$ is the identity operator on $\mathscr{D}^{+}$. Conversly every such $A$ has a domain of this type.

We now introduce the notion of abstract boundary conditions and indicate how the domain of any self-adjoint extension of $S$ can be obtained. A boundary condition is a condition on $f \in \mathscr{D}$ of the form

$$
\langle f h\rangle=0,
$$


where $h$ is a fixed function in $\mathscr{D}$. The conditions

$$
\left\langle f h_{j}\right\rangle=0, \quad j=1, \cdots, n,
$$

are said to be linearly independent if the only set of complex numbers $\alpha_{1}, \cdots, \alpha_{n}$ for which

$$
\sum_{j=1}^{n} \alpha_{j}\left\langle f h_{j}\right\rangle=0
$$

identically in $f \in \mathscr{D}$ is $\alpha_{1}=\cdots=\alpha_{n}=0$. A set of $n$ linearly independent boundary conditions $\left\langle f h_{j}\right\rangle=0, j=1, \cdots, n$, is said to be self-adjoint if $\left\langle h_{j} h_{k}\right\rangle=0, j, k=1, \cdots, n$.

The following theorem follows directly from the proof of Theorem 3 in the paper of Coddington [1].

THEOREM 3.1. If $A$ is a self-adjoint extension of $S$ with domain $\mathscr{D}_{A}$, then there exists a set of $m$ self-adjoint boundary conditions,

$$
\left\langle f h_{j}\right\rangle=0 \quad j=1, \cdots, m,
$$

such that $\mathscr{D}_{A}$ is the set of all $f \in \mathscr{D}$ satisfying these conditions. Conversly, if (3.3) is a set of $m$ self-adjoint boundary conditions, there exists a self-adjoint extension $A$ of $S$ whose domain is the set of all $f \in \mathscr{D}$ satisfying (3.3)

Let $\phi_{1}, \cdots, \phi_{m}$ and $\psi_{1}, \cdots, \psi_{m}$ be orthonormal sets for $\mathscr{D}^{+}$and $\mathscr{D}^{-}$respectively and $\left(u_{j k}\right)$ a unitary matrix representing $U$, then the $h_{j}$ are given by

$$
h_{j}=\phi_{j}-\sum_{k=1}^{m} u_{j k} \psi_{k}, \quad j=1, \cdots, m .
$$

Let $A$ be a self-adjoint operator associated with $L$ and $E(\lambda)$ the corresponding resolution of the identity. We shall show the projection $E_{\Delta}$ corrresponding to $\Delta=(a, b]$ can be expressed as an integral operator with a kernel given in terms of a basis of solutions for $L u-\lambda u=0$ and a certain spectral matrix. Our work was inspired by the treatment of E. A. Coddington [2] of the case when $A$ arises from a formal differential operator in the space $L_{2}(I), I$ an open interval. We begin by showing that the resolvent operator of $A$,

$$
R(\ell)=(A-\ell)^{-1}, \quad \operatorname{Im}(\ell) \neq 0,
$$

is an integral operator with a nice kernel. 
THEOREM 3.2. $R(\ell)$ is an integral operator with kernel $K$,

$$
R(\iota) f(z)=\iint_{|w|<1} K(z, w, \measuredangle) f(w) d u d v, \quad f \in \mathscr{\mathcal { C }}
$$

$K$ is jointly analytic in $z, \bar{w}$, and $\ell$ on the region $|z|<1,|w|<1$, $\operatorname{Im}(\iota) \neq 0$.

Moreover, $K(z, w, \iota)=\overline{K(w, z, \bar{\ell})}$ and

$$
(L-\ell) K(w, z, \iota)=K_{z}(w), \text { for fixed } z \text { and } \ell \text {. }
$$

Proof. Since $R(\ell) f(z)=\left(R(\ell) f, K_{z}\right)$ and $R^{*}(\ell)=R(\bar{\ell})$, it follows that (3.1) holds with $K(z, w, \ell)=\overline{R(\bar{\ell}) K_{z}(w)}$. Hence $K$ is analytic in $\bar{w}$ for fixed $z$ and $\iota$. That $K(z, w, \ell)=\overline{K(w, z, \bar{\ell})}$ can be seen from the following computations,

$$
K(z, w, \ell)=\overline{\left(R(\bar{\ell}) K_{z}, K_{w}\right)}=\overline{\left(K_{z}, R(\ell) K_{w}\right)}=\overline{K(w, z, \bar{\ell})} .
$$

Hence $K(z, w, \ell)$ is analytic in $z$ for fixed $w$ and $\iota$. It follows from the analyticity of $R(\ell)$ for $\operatorname{Im}(\ell) \neq 0$ that $K(z, w, \ell)=\left(R(\ell) K_{w}, K_{z}\right)$ is analytic in $\ell$ for fixed $z$ and $w$ on any region for which $\operatorname{Im}(\ell) \neq 0$. Since analyticity in each of the variables separately implies joint analyticity it only remains to verify (3.6). This follows from the fact that $K(w, z, \ell)=\overline{K(z, w, \bar{\ell})}=R(\ell) K_{z}(w)$.

We now split the kernel $K(z, w, \ell)$ into two parts one of which satisfies the homogeneous equation $(L-\ell) u=0$. Since the coefficients of $L$ are polynomials, $p_{n}$ has at most a finite number of zeros in the unit disk. Introducing radial branchcuts at these zeros, we obtain the region $\widetilde{D}$, simply connected relative to $D$, in which $p_{n}$ never vanishes. Let $z_{0} \in \widetilde{D}$, it follows from standard theorems that there exists a basis of solutions for the equation $(L-\ell) \phi=0$ such that:

(i) $\phi_{i}(\ell), i=1, \cdots, n$, are single-valued analytic functions on $\widetilde{D}$

(ii) $\phi_{i}^{(j-1)}\left(z_{0}, \ell\right)=\delta_{i j}, i, j=1, \cdots, n$,

(iii) $\phi_{i}(w, \iota), i=1, \cdots, n$, is entire in $\iota$ for each $w \in \widetilde{D}$.

THEOREM 3.3. The kernel $K(z, w, \iota)$ has the representation

$$
K(z, w, \ell)=\sum_{i, j=1}^{n} \psi_{i j}(\ell) \dot{\phi}_{i}(z, \ell) \overline{\phi_{j}(w, \bar{\ell})}+G\left(z, w, \iota^{\prime}\right),
$$

where $G(z, w, \iota)$ is entire in $\iota$ for fixed $z$ and $w$.

Proof. For fixed $z \in \widetilde{D}$ and $\operatorname{Im}(\ell) \neq 0$ it follows from (3.6) that 


$$
K(w, z, \bar{\ell})=\sum_{j=1}^{n} \psi_{j}(z, \iota) \phi_{j}(w, \bar{\ell})+\Omega(z, w, \bar{\iota}),
$$

where $\Omega(z, w, \bar{l})$ is the particular solution furnished by the variation of parameters method and is entire in $\bar{\ell}$ for fixed $z, w$. Moreover,

$$
\frac{\partial^{i-1}}{\partial w^{i-1}} \Omega\left(z, z_{0}, \bar{\ell}\right)=0, \quad i=1, \cdots, n \text {. }
$$

Now consider the differential equation $\left(L_{z}-\ell\right) K(z, w, \ell)=K_{w}(z)$, where $L_{z}$ denotes the fact that $L$ is applied with respect to $z$. Differentiating with respect to $\bar{w}$ and making use of the symmetry of $K$ we obtain

$$
\left(L_{z}-\ell\right) \frac{\partial^{j-1}}{\partial \bar{w}^{j-1}} \overline{K(w, z, \bar{\ell})}=\frac{\partial^{j-1}}{\partial \bar{w}^{j-1}} K_{w}(z), \quad j=1, \cdots, n .
$$

Using (3.8), (3.9) and the relationships

$$
\phi_{i}^{(j-1)}\left(z_{0}, \ell\right)=\delta_{i j}
$$

we obtain

$$
\left(L_{z}-\ell\right) \overline{\psi_{j}(z, \ell)}=\frac{\partial^{j-1}}{\partial \bar{w}^{j-1}} K_{z_{0}}(z)
$$

Variation of parameters yields

$$
\psi_{j}(z, \ell)=\sum_{i=1}^{n} \bar{\psi}_{i j}(\iota) \overline{\phi_{i}(z, \ell)}+\overline{\Omega_{j}(z, \ell)}, \quad j=1, \cdots, n,
$$

where the $\Omega_{j}(z, \ell)$ are entire in $\ell$ for fixed $z$ and satisfy

$$
\frac{\partial^{i-1}}{\partial z^{i-1}} \Omega_{j}\left(z_{0}, \ell\right)=0, \quad i, j=1, \cdots, n .
$$

It follows from (3.8) and (3.10) that (3.7) holds where

$$
G(z, w, \ell)=\overline{\Omega(z, w, \bar{\ell})}+\sum_{j=1}^{n} \Omega_{j}\left(z, \ell \overline{\phi_{j}(w, \bar{\ell})}\right.
$$

is entire in $\ell$ for each $z, w \in \widetilde{D}$.

Concerning the matrix $\psi=\left(\psi_{i j}\right)$ we have the following.

THEOREM 3.4. The matrix $\psi$ is analytic for $\operatorname{Im}(\ell) \neq 0, \psi^{*}(\ell)=$ $\psi(\bar{\zeta})$, and $\operatorname{Im} \psi(\ell) / \operatorname{Im}(\ell) \geqq 0$, where $\operatorname{Im} \psi=\left(\psi-\psi^{*}\right) / 2 i$.

Proof. It follows from (3.9) and (3.10) that 


$$
\psi_{i j}(\ell)=\frac{\partial^{i+j-2}}{\partial z^{i-1} \partial w^{j-1}} K\left(z_{0}, z_{0}, \iota\right), \quad i, j=1, \cdots, n
$$

and hence $\psi$ is analytic for $\operatorname{Im}(\ell) \neq 0$. Using (3.12) and the symmetry of $K$ we obtain $\psi_{i j}(\ell)=\overline{\psi_{j i}(\ell)}$.

In order to demonstrate the positivity of $\operatorname{Im} \psi(\ell) / \operatorname{Im}(\ell) \geqq 0$ we consider the functionals $l_{k}$ defined by

$$
\ell_{k}(f)=f^{(k-1)}\left(z_{0}\right), \quad f \in \mathscr{C}, k=1, \cdots, n .
$$

Since convergence in $\mathscr{H}$ implies uniform convergence on compact subsets, the $\ell_{k}$ are bounded linear functional on $\mathscr{C}$. Consequently there exist functions $K_{1}, \cdots, K_{n}$ in $\mathscr{\mathscr { C }}$ for which

$$
f^{(k-1)}\left(z_{0}\right)=\left(f, K_{k}\right),
$$

all $f$ in $\mathscr{H}$. Let $\xi_{1}, \cdots, \xi_{n}$ be any set of $n$ complex numbers and consider the function $f=\sum_{k=1}^{n} \xi_{k} K_{k}$. The inner product $(R(\ell) f, f)=$ $\sum_{i, j=1}^{n} \xi_{i} \xi_{j}\left(R(\ell) K_{i}, K_{j}\right)$. Now $R(\ell) K_{i}(z)=\left(K_{i}, K_{z}\right)$, where $K_{z \ell}(w)=$ $\overline{K(z, w, \iota)}=K(w, z, \bar{\ell})$. Consequently,

$$
R(\iota) K_{i}(z)=\overline{\frac{\partial^{i-1}}{\partial w^{i-1}} K\left(z_{0}, z, \bar{\ell}\right)},
$$

and

$$
\left(R(\ell) K_{i}, K_{j}\right)=\frac{\partial^{i+j-2}}{\partial^{i-1} \bar{w} \partial z^{j-1}} K\left(z_{0}, z_{0}, \ell\right)=\psi_{j i}(\iota)
$$

Using the resolvent equation it is not hard to see that

$$
\operatorname{Im}(R(\ell) f, f) / \operatorname{Im}(\zeta)=\|R(\ell) f\|^{2} \geqq 0
$$

and hence

$$
\sum_{i, j=1}^{n} \frac{\operatorname{Im} \psi_{j i}(\ell)}{\operatorname{Im}(\ell)} \xi_{i} \bar{\xi}_{j} \geqq 0
$$

This completes the proof.

It is shown in [2] that Theorem 3.4 implies the existence of a spectral matrix $\rho$ for the resolvent $R$.

TheOREM 3.5. The matrix $\rho$ defined by

$$
\rho(\lambda)=\lim _{\varepsilon \rightarrow+0} \frac{1}{\pi} \int_{0}^{\lambda} \operatorname{Im}(\nu+i \varepsilon) d \nu
$$

exists, is nondecreasing, and is of bounded variation on any finite interval. 
We now consider the projections $E_{4}$ corresponding to the interval $\Delta=(a, b]$. It follows from the proof of Theorem 3.2, that $E_{\Delta}$ is an integral operator with kernel $e_{\Delta}(z, w)=\overline{E_{\Delta} K_{z}(w)}$. The following theorem shows how $e_{\Delta}(z, w)$ can be described in terms of the basis $\phi_{1}, \cdots, \phi_{n}$ and the spectral matrix given by Theorem 3.5.

THEOREM 3.6. If $a$ and $b$ are continuity points of $E$ then

$$
e_{\Delta}(z, w)=\int_{\Delta} \sum_{i, j=1}^{n} \phi_{i}\left(z, \nu \overline{\dot{\phi}_{j}(w, \nu)} d \rho_{i j}(\nu),\right.
$$

where $\rho=\left(\rho_{i j}\right)$ is the spectal matrix given by Theorem 3.5.

Proof. The idea is to use the inversion formula

$$
\left.\left(E_{\Delta} f, g\right)=\lim _{\varepsilon \rightarrow+0} \frac{1}{2 \pi i} \int_{\Delta}((R \dot{\nu}+i \varepsilon) f, g)-(R(\nu-i \varepsilon) f, g)\right) d \nu,
$$

for all $f$ and $g$ in $\mathscr{X}$, $a$ and $b$ continuity points of $E_{\lambda}$. Since $E_{\Delta}$ is self-adjoint $e_{\Delta}(z, w)=\left(E_{4} K_{w}, K_{z}\right)$ and hence

$$
\begin{aligned}
e_{\Delta}(z, w) & =\lim _{\varepsilon \rightarrow+0} \frac{1}{2 \pi i} \int_{\Delta}\left\{\left(R(\nu+i \varepsilon) K_{w}, K_{z}\right)-\left(R(\nu-i \varepsilon) K_{w}, K_{z}\right)\right\} d \nu . \\
& =\lim _{\varepsilon \rightarrow+0} \frac{1}{2 \pi i} \int_{\Delta} K(z, w, \nu+i \varepsilon)-K(z, w, \nu-i \varepsilon) d \nu .
\end{aligned}
$$

For $z, w \in \widetilde{D}$, this becomes

$$
\begin{aligned}
\lim _{\varepsilon \rightarrow+0} & \frac{1}{2 \pi i} \int_{\Delta} \sum_{i, j=1}^{n} \psi_{i j}(\nu+i \varepsilon) \phi_{i}(z, \nu+i \varepsilon) \overline{\phi_{j}(w, \nu-i \varepsilon)} \\
& -\psi_{i j}(\nu-i \varepsilon) \phi_{i}(z, \nu-i \varepsilon) \overline{\phi_{j}(w, \nu+i \varepsilon)} d \nu \\
& +\lim _{\varepsilon \rightarrow+0} \frac{1}{2 \pi i} \int_{\Delta} G(z, w, \nu+i \varepsilon)-G(z, w, \nu-i \varepsilon) d \nu .
\end{aligned}
$$

Since $G(z, w, \ell)$ is entire in $\ell$ the later integral tends to zero as $\varepsilon \rightarrow+0$.

We now rewrite the first integrand as

$$
\begin{aligned}
& \sum_{i, j=1}^{n}\left[\psi_{i j}(\nu+i \varepsilon)-\psi_{i j}(\nu-i \varepsilon)\right] \phi_{i}(z, \nu) \overline{\phi_{j}(w, \nu)}+ \\
& \left.\sum_{i, j=1}^{n} \psi_{i j}(\nu+i \varepsilon)\left[\phi_{i}(z, \nu+i \varepsilon) \overline{\phi_{j}(w, \nu-i \varepsilon)}-\phi_{i}(z, \nu) \overline{\phi_{j}(w, \nu}\right)\right]+ \\
& \sum_{i, j=1}^{n} \psi_{i j}(\nu-i \varepsilon)\left[\phi_{i}(z, \nu) \overline{\phi_{j}(w, \nu)}-\phi_{i}\left(z, \nu-i \varepsilon \overline{\phi_{j}(w, \nu+i \varepsilon)}\right]\right.
\end{aligned}
$$

and denote the three sums by $I_{1}(\nu, \varepsilon), I_{2}(\nu, \varepsilon)$, and $I_{3}(\nu, \varepsilon)$ respectively. Consider $I_{1}(\nu, \varepsilon)$, 


$$
\lim _{\varepsilon \rightarrow+0} \frac{1}{2 \pi i} \int_{\Delta} I_{1}(\nu, \varepsilon) d \nu=\lim _{\varepsilon+0} \frac{1}{\pi} \int_{\Delta} \sum_{i, j=1}^{n} \operatorname{Im} \psi_{i j}(\nu+i \varepsilon) \phi_{i}(z, \nu) \overline{\phi_{j}(w, \nu)} d \nu
$$

Now

$$
\rho(\lambda)=\lim _{\varepsilon \rightarrow+0} \frac{1}{\pi} \int_{\Delta} \operatorname{Im} \psi(\nu+i \varepsilon) d \nu
$$

and it follows from a theorem of Helly that

$$
\lim _{\varepsilon \rightarrow+0} \frac{1}{2 \pi i} \int_{\Delta} I_{1}(\nu, \varepsilon) d \nu=\int_{\Delta} \sum_{i, j=1}^{n} \phi_{i}(z, \nu) \overline{\phi_{j}(w, \nu)} d \rho_{i j}(\nu) \text {. }
$$

As is shown in [2] we have the following estimate

$$
\sum_{i, j=1}^{n} \int_{\Delta}\left|\psi_{i j}(\nu \pm i \varepsilon)\right| d \nu=O\left(\log \frac{1}{\varepsilon}\right) \quad(\varepsilon \rightarrow+0) .
$$

Since the $\phi_{i}(z, \ell)$ are entire in $\ell$ for fixed $z$ there exists a constant $M>0$ such that for $\varepsilon$ sufficiently small

$$
\left|\phi_{i}(z, \nu+i \varepsilon) \overline{\phi_{j}(w, \nu-i \varepsilon)}-\phi_{i}(z, \nu) \overline{\phi_{j}(w, \nu)}\right|<M \varepsilon
$$

for all $\nu \in \Delta$.

Combining (3.15) and (3.16) we see that

$$
\frac{1}{\pi} \int_{\Delta} I_{2}(\nu, \varepsilon) d \nu=O\left(\varepsilon \log \frac{1}{\varepsilon}\right) \quad(\varepsilon \rightarrow+0),
$$

which tends to zero as $\varepsilon \rightarrow+0$. A similar result holds for

$$
\frac{1}{\pi} \int_{\Delta} I_{3}(\nu, \varepsilon) d \nu \text {. }
$$

Consequently we have

$$
e_{\Delta}(z, w)=\int_{\Delta} \sum_{i, j=1}^{n} \phi_{i}(z, \nu) \overline{\phi_{j}(w, \nu)} d \rho_{i j}(\nu) .
$$

The author wishes to express his gratitude to Professor Earl Coddington for his encouragement and guidance in this work.

\section{BIBLIOGRAPHY}

1. E. A. Coddington, The spectral representation of ordinary self-adjoint differential operators, Ann. of Math. 60 (1954), 192-211.

2. - Generalized resolutions of the identity for symmetric ordinary differential operators, Ann. of Math. 68 (1958), 378-392.

3. N. Dunford and J. Schwartz, Linear Operators, Part II, Interscience Publishers, 1963. 
Received February 20, 1970. This work is part of a doctoral dissertation written at the University of California at Los Angeles under Professor Earl Coddington and supported in part by NSF Grant GP-3594.

University of California, Los ANgeles

AND

San Diego State College 



\section{PACIFIC JOURNAL OF MATHEMATICS}

\section{EDITORS}

\author{
H. SAMELSON \\ Stanford University \\ Stanford, California 94305

\section{Richard Pierce} \\ University of Washington \\ Seattle, Washington 98105
}

J. DugundJI

Department of Mathematics

University of Southern California

Los Angeles, California 90007

RichaRd ARENS

University of California

Los Angeles, California 90024

\section{ASSOCIATE EDITORS}
E. F. BECKENBACH
B. H. NeUMANN
F. WOLE
K. YoshidA

\section{SUPPORTING INSTITUTIONS}

\author{
UNIVERSITY OF BRITISH COLUMBIA \\ CALIFORNIA INSTITUTE OF TECHNOLOGY \\ UNIVERSITY OF CALIFORNIA \\ MONTANA STATE UNIVERSITY \\ UNIVERSITY OF NEVADA \\ NEW MEXICO STATE UNIVERSITY \\ OREGON STATE UNIVERSITY \\ UNIVERSITY OF OREGON \\ OSAKA UNIVERSITY \\ UNIVERSITY OF SOUTHERN CALIFORNIA
}

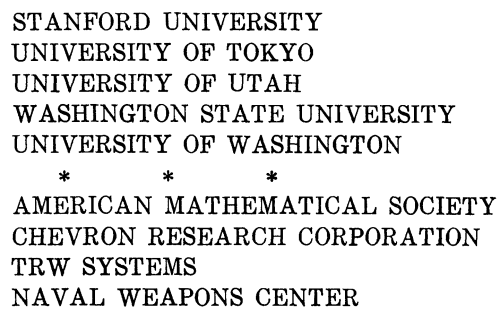

The Supporting Institutions listed above contribute to the cost of publication of this Journal, but they are not owners or publishers and have no responsibility for its content or policies.

Mathematical papers intended for publication in the Pacific Journal of Mathematics should be in typed form or offset-reproduced, (not dittoed), double spaced with large margins. Underline Greek letters in red, German in green, and script in blue. The first paragraph or two must be capable of being used separately as a synopsis of the entire paper. The editorial "we" must not be used in the synopsis, and items of the bibliography should not be cited there unless absolutely necessary, in which case they must be identified by author and Journal, rather than by item number. Manuscripts, in duplicate if possible, may be sent to any one of the four editors. Please classify according to the scheme of Math. Rev. Index to Vol. 39. All other communications to the editors should be addressed to the managing editor, Richard Arens, University of California, Los Angeles, California, 90024.

50 reprints are provided free for each article; additional copies may be obtained at cost in multiples of 50 .

The Pacific Journal of Mathematics is published monthly. Effective with Volume 16 the price per volume (3 numbers) is $\$ 8.00$; single issues, $\$ 3.00$. Special price for current issues to individual faculty members of supporting institutions and to individual members of the American Mathematical Society: $\$ 4.00$ per volume; single issues $\$ 1.50$. Back numbers are available.

Subscriptions, orders for back numbers, and changes of address should be sent to Pacific Journal of Mathematics, 103 Highland Boulevard, Berkeley, California, 94708.

PUBLISHED BY PACIFIC JOURNAL OF MATHEMATICS, A NON-PROFIT CORPORATION

Printed at Kokusai Bunken Insatsusha (International Academic Printing Co., Ltd.), 7-17, Fuj̣imi 2-chome, Chiyoda-ku, Tokyo, Japan. 


\section{Pacific Journal of Mathematics}

\section{Vol. 35, No. $2 \quad$ October, 1970}

Valentin Danilovich Belousov and Palaniappan L. Kannappan, Generalized Bol functional equation .................................... 259

Charles Morgan Biles, Gelfand and Wallman-type compactifications ........... 267

Louis Harvey Blake, A generalization of martingales and two consequent convergence theorems .................................... 279

Dennis K. Burke, On p-spaces and $w \Delta$-spaces..................... 285

John Ben Butler, Jr., Almost smooth perturbations of self-adjoint operators . . . . . . 297

Michael James Cambern, Isomorphisms of $C_{0}(Y)$ onto $C(X) \ldots \ldots \ldots \ldots \ldots . \ldots 307$

David Edwin Cook, A conditionally compact point set with noncompact closure ... 313

Timothy Edwin Cramer, Countable Boolean algebras as subalgebras and homomorphs .........................................

John R. Edwards and Stanley G. Wayment, A v-integral representation for linear operators on spaces of continuous functions with values in topological vector spaces.............................................

Mary Rodriguez Embry, Similarities involving normal operators on Hilbert

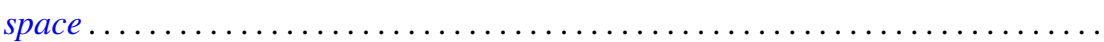

Lynn Harry Erbe, Oscillation theorems for second order linear differential

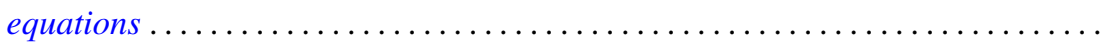

William James Firey, Local behaviour of area functions of convex bodies .......... Joe Wayne Fisher, The primary decomposition theory for modules ..............

Gerald Seymour Garfinkel, Generic splitting algebras for Pic ..................

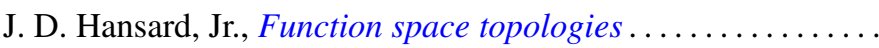

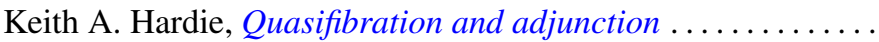

G. Hochschild, Coverings of pro-affine algebraic groups ...........

Gerald L. Itzkowitz, On nets of contractive maps in uniform spaces ..

381

389

399

417

Melven Robert Krom and Myren Laurance Krom, Groups with free nonabelian subgroups....................................

James Robert Kuttler, Upper and lower bounds for eigenvalues by finite differences ......................................

Dany Leviatan, A new approach to representation theory for convolution transforms . . .

Richard Beech Mansfield, Perfect subsets of definable sets of real numbers ...

Brenda MacGibbon, A necessary and sufficient condition for the embedding of a

Lindelof space in a Hausdorff $\mathscr{H} \sigma$ space ..................

David G. Mead and B. D. McLemore, Ritt's question on the Wronskian ....

Edward Yoshio Mikami, Focal points in a control problem .....

Paul G. Miller, Characterizing the distributions of three independent n-dimensional random variables, $X_{1}, X_{2}, X_{3}$, having analytic characteristic functions by the joint distribution of $\left(X_{1}+X_{3}, X_{2}+X_{3}\right)$. . .

P. Rosenthal, On the Bergman integral operator for an elliptic partial differential equation with a singular coefficient....

Douglas B. Smith, On the number of finitely generated $O$-group 